\title{
La dimensión comunitaria en Servicios Sociales. Una aproximación a su tratamiento en barrios vulnerables
}

\author{
Community Standing in Social Services: \\ Focus on its Importance in Vulnerable Neighborhoods
}

\author{
Germán Jaraíz Arroyo \\ Universidad Pablo de Olavide \\ gjararr@upo.es
}

La protección social del futuro o será colectiva, o no será. Zygmunt Bauman

Recibido: 03/10/2011

Revisado: 24/11/2011

Aceptado: 04/11/2011

Disponible on line: 15/02/2012

\section{Resumen}

Este artículo actualiza el tratamiento que el enfoque comunitario ha tenido en los Servicios Sociales modernos. En la primera parte se narra el tratamiento que el asunto ha tenido desde que se configuron estos dispositivos hasta el tiempo actual. En un segundo momento se detiene en la aplicación práctica del trabajo comunitario, utilizando como soporte las percepciones y discursos de los profesionales de Servicios Sociales de los barrios vulnerables de una gran ciudad andaluza. En la parte final indaga en las lógicas que han caracterizado la intervención comunitaria, identificando los límites y aportaciones que, orientadas a su mejora, hacen los profesionales.

Palabras clave: servicios sociales, comunidad, intervención social, trabajo social.

\begin{abstract}
This article analyzes the importance that the community-based approach has taken in modern Social Services. In the first part, the evolution that the subject has experienced since configuration of these mechanisms up to the current time is described. The second part focuses on the practical implementation of community work, using the perceptions and speeches of Social Service professionals in vulnerable neighborhoods in a large city of the Andalusian region as supporting evidence. The final part plumbs the mentality which characterizes community intervention while identifying the limits and contributions of professionals seeking its improvement.
\end{abstract}

Keywords: Social Services, Community, Social intervention, Social work.

Referencia normalizada: Jaraíz Arroyo, G. (2012): «La dimensión comunitaria en Servicios Sociales. Una aproximación a su tratmiento en barrios vulnerables». Cuadernos de Trabajo Social, 25(1): 113-124.

Sumario: Introducción. 1. El encuentro entre comunidad y servicio social. Antecedentes y primeras formas, aproximaciones a un enfoque globalizador. 2. El asentamiento de la concepción modernizadora. La reducción del enfoque comunitario. 3. Los efectos sobre los barrios vulnerables. 4. La innovación y el retorno. La planificación comunitaria integral y gobernanza local. 5. Dos lógicas en busca de dialógica. 6. Pistas de renovación desde el discurso profesional. Recuperar el Trabajo Social en Servicios Sociales. 7. A modo de conclusión. 8. Referencias bibliográficas.

\section{Introducción}

Los Servicios Sociales modernos aparecen insertos en el proyecto de sociedad democrática. Nacen con el encargo de superar un modelo de acción social altamente asociado a la beneficencia, muy fragmentado, poco operativo y predominantemente discrecional; para posibilitar nuevas dinámicas de intervención social 
basadas en una actuación preventiva y de desarrollo comunitario que huya del asistencialismo; y priorice las relaciones de cooperación (Subirats et al, 2007, p. 19). Esta es la ruta por la que son encauzados, como dispositivos a los que se asigna el encargo de mejorar la calidad de vida de la ciudadanía, promoviendo el bienestar social. Aunque es cuestión sabida, resulta procedente tomar aquí como guía esta idea para no perder el rumbo, en momentos como el presente en los que el vértigo de la turbulencia parece dejar en segundo plano el sentido del trayecto.

La referencia a lo comunitario viene por tanto inserta en el $A D N$ de los Servicios Sociales. Este trabajo aborda con ánimo descriptivo el desarrollo general de esta idea, deteniéndose con mayor detalle en su efecto práctico sobre una tipología concreta de espacio interventor en el que el enfoque comunitario $^{1}$ resulta especialmente relevante por necesario: el barrio vulnerable. Para ello utilizo como soporte dos trabajos de investigación ${ }^{2}$ que se centran en las prácticas y discursos de los profesionales que trabajan en distintos centros de Servicios Sociales comunitarios, que operan en barrios vulnerables de una gran ciudad andaluza.

\section{El encuentro entre comunidad y servicio social. Antecedentes y primeras formas, aproximaciones a un enfoque globalizador ${ }^{3}$}

A mediados de los años 1960, Víctor Pérez Díaz (1965) realiza en la revista Anales de Economía un análisis sobre las políticas de desarrollo económico en las zonas rurales. En su trabajo advierte de la existencia de un movimiento alternativo al modelo de desarrollo oficial promovido por el Estado. Es, para el autor, un movimiento interesante al que se está prestando muy escasa atención. Este modelo posee dos características diferenciales: 1) está promocionado por profesionales (entre ellos asistentes sociales) y por entidades diversas; y 2) se concibe como un servicio social. Pérez Díaz identifica como emergen nuevos modos de hacer, a la sombra de algunas de las iniciativas de desarrollo pensadas desde lo económico, que incorporan la matriz social, uniendo las ideas de desarrollo comunitario ${ }^{4}$ y servicio social. Un año después, Marchioni (1966) publica en la revista Documentación Social la que, tal vez es la primera aproximación teórico-metodológica al desarrollo comunitario en entornos urbanos en nuestro país. Se trata de una intervención de servicio social guiada por un conjunto de criterios como: el protagonismo de

\footnotetext{
${ }^{1}$ Formalmente el discurso sobre lo comunitario viene asociado en Servicios Sociales al territorio y al espacio que comparte la gente. La comunidad aquí referida nombra una escala de relaciones que, trascendiendo un nivel primario (familia y amigos), resulta abarcable para la intervención, tanto socialmente (estatus asimilables), como especialmente (proximidad física). Lo que aquí denomino enfoque comunitario se refiere al conjunto de criterios y lógicas que orientan la práctica interventora en este tipo de espacios.

${ }^{2}$ Ambas investigaciones se centran en el estudio de las prácticas y discursos de los profesionales de Servicios Sociales comunitarios acerca del sentido de su intervención. Han sido realizadas entre mayo de 2006 y febrero de 2011 por el Grupo de Investigación, GISAP, de la Universidad Pablo de Olavide y dirigidas por el autor este texto. La primera de ellas trabaja sobre los discursos de los profesionales de cuatro centros de Servicios Sociales que operan en barriadas calificadas como zonas con especiales necesidades de transformación social. La segunda se orienta a la descripción de las prácticas comunitarias en uno de estos centros. Ambas investigaciones comparten un mismo diseño metodológico. Son trabajos cualitativos, de naturaleza participada (soportados en la discusión horizontal entre investigadores y profesionales). El proceso de investigación se ha ordenado en torno a los siguientes pasos: 1) Observar prácticas y recoger discursos sobre las mismas (de diferentes actores); 2) Codificar y sistematizar la información mediante procedimientos explícitos y reconocidos. 3) Analizarla, con el debido contraste teórico, en el equipo de investigación. 4) Devolver el conocimiento a los protagonistas para discutir y reformular participativamente el análisis. 5) Identificar criterios y vías de aplicación.

${ }^{3}$ Esto que llamo enfoque globalizador representa una lógica específica de enfoque comunitario.

${ }^{4}$ Según José Luis Malagón Bernal (1999), el concepto de desarrollo comunitario surge después de la Segunda Guerra Mundial y se entiende como una actuación de base eminentemente económica. Se dirige en sus orígenes a países colonizados en condiciones socioeconómicas muy deterioradas y a los países en vías de desarrollo en general. Este esquema de intervención será aplicado en España, en partir de los años 1950, a zonas deprimidas rurales y urbanas ( p. 122).
} 
la comunidad, la relación entre la dinamización de la economía local y la generación de servicios y recursos para el bienestar, o el carácter interdependiente de la intervención necesaria.

Aunque estos enfoques que unen desarrollo comunitario y servicio social no van a generalizarse en este tiempo, sí es cierto que durante la década siguiente van a llevarse a cabo diferentes experiencias significativas en esta senda en distintos puntos de España ${ }^{5}$. Josefa Fombuena (2000) apunta que en este contexto se va a producir lo que denomina el primer consenso del Trabajo Social, en el que los Servicios Sociales son evocados (por deseados aún) como un dispositivo institucional con capacidad para posibilitar: 1) el desarrollo técnico y metodológico de la disciplina; 2) la consolidación de la visión del profesional como agente de cambio; y 3) la búsqueda de un enfoque global de intervención. Las trabajadoras sociales, primeras pensadoras de este modelo de Servicios Sociales, entienden que para hacerlo posible, dichos dispositivos habrían de cubrir un conjunto de funcionalidades en lo comunitario (Consejo General de Trabajadores Sociales, 1986), entre las que destacan:

- Abarcar a toda la población, superando el encasillamiento profesional.

- Integrar acciones preventivas y promocionales, superando las meramente recuperadoras.

- Potenciar la gestión local, reforzando la presencia de entidades locales de Servicios Sociales.

- Ser puerta de entrada a la red de servicios y clave en la estructuración de la intervención.

- Ser dinamizadores de las relaciones sociales y de la participación.

- Implicarse con otras áreas, como la económica, en la generación de dinámicas de desarrollo local.

Si se mira a este conjunto de cometidos queda patente la visión de unos Servicios Sociales (espacio institucional) y un Trabajo Social (sustento metodológico-epistemológico), ubicados estratégicamente en el interface de dos metas: el bienestar social y el desarrollo local.

A esta concepción se acogerán luego, al menos formalmente, los diferentes marcos reguladores en los sistemas autonómicos, que dan lugar a una articulación de los Servicios Sociales en dos niveles operativos: uno básico o de proximidad, que atiende a lo común, y otro especializado para situaciones y problemas específicos. El nivel básico será denominado de distinta forma, según las diversas leyes: servicios sociales comunitarios, de atención primaria, de atención de base. En esta pluralidad de denominaciones, que ha persistido en las legislaciones de segunda y tercera generación (Vilá, 2010), se apuntan dos esquemas preliminares: uno que adopta la denominación que prioriza el contexto en el que se encuadra la intervención, como es el caso de la denominación servicios sociales comunitarios; y el otro, que lo hace atendiendo a la posición jerárquica que ocupa el dispositivo dentro de la estructura interna del sistema. A esta forma se corresponden las denominaciones servicios sociales de atención social de base o primaria.

Ya en el tratamiento legislativo primero parecen asentarse aproximaciones diferentes a la dimensión comunitaria que no son ajenas, como se verá después, al modo de pensar el modelo de intervención social.

\subsection{La aparición de los primeros centros de Servicios Sociales comunitarios. Bienestar y desarrollo}

Según los presupuestos anteriores se generaliza, a principios de los años 1980, la aparición de delegaciones e institutos dedicados a la promoción de los Servicios Sociales comunitarios en las grandes ciudades. A estas estructuras se incorporan profesionales que reflexionan, debaten, discuten e inventan los Servicios Sociales comunitarios, como expresa el testimonio ${ }^{6}$ de una trabajadora social testigo del momento:

\footnotetext{
${ }^{5}$ Véase Casado, 2007, pp. 101-146.

${ }^{6}$ Hay que aclarar aquí que los discursos que se citan a lo largo de este trabajo han sido obtenidos por dos vías: la primera mediante la entrevista semiestructurada. Se han realizado un total de 27 destinadas a diferentes actores (técnicos, ciudadanos, políticos), en algunos casos esta entrevista ha sido de carácter gru-
} 
Nosotras éramos casi todas trabajadoras sociales, muchas habíamos terminado la carrera en la transición, en la Escuela se hablaba de la «reconceptualización», del carácter transformador y de la dimensión política del Trabajo Social, pero nadie nos había hablado de Servicios Sociales. El ayuntamiento (de Sevilla) nos contrató a cinco para que organizásemos los Servicios Sociales comunitarios y tuvimos que aprender, viendo lo que había hecho otra gente y también que inventarlo.

En este contexto aparecen en algunas ciudades los primeros centros de Servicios Sociales comunitarios. En este primer momento estos dispositivos son ideados como espacios de proximidad para promover servicios personales, pero también como lugares para la conexión con el tejido local; y concebidos simultáneamente en clave de servicio y acción comunitaria (Consejo General de Trabajo Social, 1986). Se asienta un patrón de intervención en lo local en el que lo comunitario parece tener dos cometidos simultáneos: 1) atender un objeto específico de acción (la comunidad), y 2) conectarlo con las otras dimensiones del modelo de interventor de estos servicios (la dimensión individual y la grupal).

Cuando empecé a trabajar (año 1985) casi no había recursos, aún teníamos que gestionar las ayudas de Padrón Municipal de Beneficencia. Este fue el encargo que nos hicieron, pero vimos que nuestro papel estaba en la comunidad. Promovimos una plataforma de recursos de toda la zona, implicamos a los colegios, al centro de salud, asociaciones de vecinos, la Junta de Distrito (...). Desde ahí hicimos el primer estudio del barrio, nos tocó dinamizar todo este trabajo conjunto, nos encontrábamos en el Centro (...) Tratamos de cambiar el asistencialismo del Padrón por desarrollo comunitario (...) fue un momento emocionante, yo sentía que hacía trabajo social (Trabajadora Social de un centro de Servicios Sociales comunitario).

Más allá del tinte nostálgico del relato, el anterior discurso muestra un tipo de posicionamiento característico de los Servicios Sociales comunitarios de ese tiempo inicial en el que no existía siquiera una legislación básica de Servicios Sociales y los profesionales, trabajadoras sociales casi exclusivamente en este caso, dedican parte de sus capacidades y energías a sortear la lógica establecida para favorecer la apertura de nuevas vías de intervención marcadas por la intención globalizadora.

\section{El asentamiento de la concepción moder- nizadora. La reducción del enfoque comuni- tario}

Poco después, ya en el ocaso de la década de 1980, el anterior enfoque irá modificándose hasta ser sustituido por un nuevo discurso. En este segundo momento, en el que se produce el despliegue generalizado en el conjunto de municipios, los comunitarios son considerados sobre todo como una estructura para aproximar al ciudadano un conjunto de bienes sociales básicos (Arriba, 2008). Bajo criterios como: universalización, descentralización, prevención y responsabilidad pública, se trata de superar la discrecionalidad y dispersión de etapas anteriores, y concentrar las energías en la promoción de una cartera, más o menos preconfigurada, de servicios de lógica personalizada. Es la gran prioridad del momento.

En esta etapa el discurso del desarrollo comunitario se irá dejando a un lado, por considerarse como una propuesta excesivamente politizada y asociada con frecuencia a posiciones reconceptualizadoras, propias de una transición política que se desea cerrar de modo definitivo. Emerge entonces una concepción modernizadora (IOE, 1990) en la que el dinamismo de lo local se expresa con un nuevo enunciado, más técnico y aséptico: la intervención comunitaria. El nuevo concepto se instala en la cotidianidad de los profesionales. Más allá de cambios terminológicos, lo destacable es que en este momento, al tiempo que se incrementan las prestaciones se resta relevancia en la práctica al valor transversal del enfoque comunitario, que será relegado de método articulador a una mera técnica de intervención.

A ello contribuyen también los distintos instrumentos financieros, que favorecerán la

pal, siempre con pocos informantes (nunca más de tres). La otra fuente, la más relevante, ha sido la conversación no programada como entrevista, este registro ha sido posible mediante la presencia intensa del investigador en el Centro y la participación del mismo espacios cotidianos (conversaciones en el pasillo, reuniones de trabajo, etc.). 
conformación del nivel comunitario del sistema en torno a cuatro servicios básicos ${ }^{7}$, y focalizarán la comentada intervención comunitaria en uno de ellos: el servicio de cooperación social o colaboración social). Este servicio asume, como tarea más frecuente, la coordinación técnica con determinadas asociaciones y entidades sociales que colaboran con la Administración local del bienestar. El todo sobre el que se pensó el nivel básico se iba a concretar, por cuestiones de operatividad, en una parte, un programa o un servicio más, dentro del catálogo de los servicios comunitarios.

La incuestionable mejora de recursos, prestaciones y contenidos de atención personal (en el rol de provisores de bienes) se torna en merma en la definición un proyecto cívico compartido y de las lógicas de intervención de conjunto. Lo relacional comunitario queda restringido principalmente a relaciones de interés fáctico. Así expresaba la vivencia de este proceso una trabajadora social:

Aquí ahora (2008) no se hace el trabajo comunitario de antes. Nos dedicamos (ahora) a gestionar recursos y servicios, sobre todo para la atención a la dependencia y para menores en riesgo. El contacto con eso que llamamos la comunidad consiste más que nada en la colaboración con las asociaciones, con las parroquias y con algunas ONG que participan en la convocatoria de ayuda del Ayuntamiento. Este es el único trabajo comunitario que hago: coordinarme con entidades que colaboran en la gestión de los casos.

En realidad, esta tendencia modernizadora responde a un modelo de organización de la intervención asentada en la visión de que el todo es resultado de la suma de cada parte (Renes et al, 2007). La acción interventora precisará así dos condiciones: 1) que cada entidad presente en lo local se encargue de un ámbito específico de necesidades (educativo, sanitario, social...); y 2) que, de puertas adentro, cada cual establezcan el conjunto de programas o servicios que atiendan a cada necesidad concreta. Como expresa el discurso anterior, la in- tervención profesional en este contexto se concentra en la gestión de la demanda. Este constreñimiento opera de modo reductor en las relaciones Servicios Sociales-comunidad, debilita la conexión de tipo estratégico y concentra la intervención profesional en relaciones de naturaleza táctica, orientadas a resolver problemas concretos que apenas se ponen en relación con el conjunto de los procesos que afectan a los sujetos que comparten un espacio existencial.

\section{Los efectos sobre los barrios vulnerables} Palmo arriba, palmo abajo: estos son los esquemas que han venido operando en Servicios Sociales comunitarios. En el contexto urbano investigado, los profesionales piensan que este modelo contiene utilidades para la intervención en aquellos barrios más normalizados. Sin embargo, en los barrios vulnerables de la ciudad, lugares en los que la confluencia de múltiples factores de fragilidad social y el deterioro relacional hace que exista un todo diferente a la suma de las partes, las debilidades de la dinámica se muestran de modo intenso.

Por un lado, las grietas de los dinamismos de conjunto (social, sanitario, educativo, laboral y cívico) ponen de manifiesto la escasa capacidad de los diversos servicios, concebidos en clave de bienestar, para frenar el deterioro social de estos espacios. Por el otro, factores como la juventud relativa de los comunitarios (último sistema de bienestar en incorporarse), o la indefinición de sus contenidos, dotan de una elevada porosidad a estos dispositivos. Esta cuestión hace que el conjunto de problemas y demandas no resueltas por la red pública de bienestar terminen desviados con una virtual naturalidad hacia estos dispositivos. Un profesional lo expresa así: «aquí, los problemas que no son de nadie, son de los Servicios Sociales comunitarios»».

A este enrocamiento en lo asistencial ha contribuido también la discrecionalidad política en no pocas dosis. En el plano estrictamente legislativo, el marco legal que da sentido de conjunto al sistema se estableció, para el caso

\footnotetext{
${ }^{7}$ Me refiero a los servicios encargados de las prestaciones básicas de Servicios Sociales comunitarios: información, valoración y orientación; Ayuda a Domicilio; Convivencia y Reinserción y Cooperación Social a cuya consolidación ha contribuido el Plan Concertado de Prestaciones Básicas de Servicios Sociales en las Corporaciones Locales.
} 


\begin{tabular}{|c|c|c|}
\hline $\begin{array}{l}\text { Organización base . } \\
\text { Cometidos iniciales }\end{array}$ & $\begin{array}{c}\text { Desarrollos } \\
\text { Encargo más relevantes }\end{array}$ & $\begin{array}{c}\text { Demandas a la intervención } \\
\text { profesional }\end{array}$ \\
\hline $\begin{array}{l}\text { Servicio de Información } \\
\text { y Orientación (SIO). } \\
\text { Servicio de Ayuda } \\
\text { a Domicilio (SAD). } \\
\text { Servicio de Convivencia } \\
\text { y Reinserción (CORE). } \\
\text { Servicio } \\
\text { de Cooperación Social } \\
\text { (COSO). }\end{array}$ & $\begin{array}{l}\text { Apoyo a diferentes políticas } \\
\text { específicas (discapacidad, } \\
\text { mayores, toxicomanías). } \\
\text { Protección (menores, } \\
\text { familias, discapacitados). } \\
\text { Gestión de garantía de } \\
\text { rentas mínimas. } \\
\text { Gestión y prestaciones } \\
\text { asociadas a la Ley de } \\
\text { dependencia. } \\
\text { Intervención en Zonas } \\
\text { Necesitadas de } \\
\text { Transformación } \\
\text { Social (barrios } \\
\text { vulnerables). }\end{array}$ & $\begin{array}{l}\text { Informar a los ciudadanos de todo } \\
\text { recurso social. } \\
\text { Prestar servicios directos (SAD, talleres } \\
\text { grupos de autoayuda...). } \\
\text { Atender toda emergencia social de la } \\
\text { comunidad. } \\
\text { Acompañar procesos personales y/o } \\
\text { familiares de integración. } \\
\text { Proteger situaciones de desamparo. } \\
\text { Informar técnicamente y/o pericialmente } \\
\text { en situaciones vulnerables (sistema } \\
\text { judicial, educativo, arraigos, etc.). } \\
\text { Impulsar y coordinar redes (con entidades } \\
\text { colaboradoras, etc.). }\end{array}$ \\
\hline
\end{tabular}

Cuadro 1. Contenidos de los servicios sociales en los barrios vulnerables.

estudiado, con la Ley 2/98, de Servicios Sociales de Andalucía. Posteriormente han ido apareciendo legislaciones en diferentes materias (drogodependencias, protección de menores, garantía de rentas, dependencia, etc.). Todas ellas asignan nuevos encargos a los comunitarios, pero sin apenas previsión del impacto sobre sus contenidos centrales. La ausencia de una legislación de conjunto en Servicios Sociales más moderna (a diferencia de la mayoría de otras comunidades autónomas), ha contribuido en Andalucía al desorden del escenario actual y desdibujado, aún más si cabe, el deseable enfoque comunitario.

Al tiempo que se debilita el liderazgo inicial de los Servicios Sociales sobre lo comunitario, para especializarse en la prestación de servicios personales, emergen los nuevos y crecientes encargos venidos de fuera (Junta, juzgados, etc.) y de dentro (colegios, centros de salud, etc.) que contribuyen a difuminar el sentido inicial de Servicios Sociales y refuerzan su rol como agencia asistencial. Así lo expresa uno de los profesionales de estos centros: «cada vez atendemos menos a la gente y más a otras instituciones, el 40 por ciento de los expedientes (casos) que tenemos entre manos son peticiones externas, informes, arraigos, trámites $(. .$.$) y la cosa va en aumento».$
Esta dinámica, recogida de forma sintética en el cuadro 1, incrementa de forma creciente el conjunto de demandas sobre sus profesionales, y los condena a focalizar la práctica totalidad de sus energías en la gestión de las mismas.

Los desenfoques en torno al enfoque comunitario se producen por tanto en dos movimientos que se desarrollan de modo casi simultáneo: 1) Los Servicios Sociales optan por un modelo de diferenciación, frente a un enfoque de globalidad (siguiendo la tendencia del momento). 2) La debilidad inherente a los mismos y la ausencia de espacios revisión entre los diferentes actores condenan a los Servicios Sociales comunitarios de estos barrios a actuar en un casi de permanente estado de emergencia social.

Resulta paradójico que, al tiempo que se limita el papel de estos dispositivos como nodo en lo comunitario, el desentendimiento de tal función provoca una especie de efecto boomerang que revierte hacia los mismos una insaciable cantidad de nuevos encargos e incorpora crecientes dificultades para el contacto con los sujetos.

Ante este panorama, las administraciones han introducido diversas medidas especiales de apoyo a los Servicios Sociales comunitarios de 
estos barrios (que en el ámbito andaluz reciben la denominación de zonas urbanas necesitadas de transformación social). Tales medidas van a regirse por el principio a más problemas sociales, más recursos, lo que ha permitido a los Servicios Sociales disponer de más financiación y más personal para sus programas. Sin embargo, diversos trabajos (Rosa, 2010; Jaraíz, 2004) dejan patente que el incremento de recursos tampoco ha tenido un efecto destacable en la reversión de la tendencia exclusógena de estos barrios. Los profesionales entienden que el problema aquí no es sólo de falta de medios (que también), sino de las incoherencias del modelo de intervención:

Este barrio es un saco sin fondo, todos los recursos que pongas son pocos, el problema no es sólo esto, es también de falta de sentido de lo que se hace, no hay una visión común, mientras esto no exista la cosa seguirá igual.

\section{La innovación y el retorno. La Planifica-} ción comunitaria integral y gobernanza local En el tiempo reciente las limitaciones de esta dinámica de intervención adquieren un cierto efecto mediático, en algunos barrios de esta y otras ciudades andaluzas el movimiento vecinal llega a denunciar la insostenibilidad e incoherencia del modelo de intervención en su conjunto ${ }^{8}$. Esta situación provoca una reacción política de Servicios Sociales y de otras áreas del bienestar que va facilitar la puesta en marcha de algunas experiencias innovadoras que marcan un tercer movimiento en torno al tratamiento de lo comunitario en Servicios Sociales.

El instrumento de renovación frecuente en el caso andaluz ha sido el Plan comunitario integral. Mediante la restauración de esta vieja herramienta (la planificación local), se trata de revertir el modelo de intervención social apostado por dos criterios centrales:

1) Reticularidad, entendida como incardinación o ensamblaje del conjunto de políticas, actuaciones y dispositivos específicos que actúan en el territorio (educativos, de empleo, sanitarios, de vivienda, sociales, de seguridad, etc.) en torno a una estrategia global de acción;
2) Participación en la gobernanza, entendida como dinámica relacional de naturaleza dialógica, que incorpora a las tareas de diseño, ejecución y evaluación de la intervención social a los diversos actores, tanto en sentido vertical (estatales, autonómico y locales), como horizontal (entidades públicas, vecinales, del tercer sector y privadas).

Estas nuevas formas de presencia obligan a los comunitarios a resituarse como un actor inserto en un espacio reticular amplio, en el que han de intervenir como co-protagonistas del conjunto de iniciativas de planificación integral en el territorio. Se recupera en gran parte la idea fundadora de desarrollo del territorio, priorizando las interconexiones al mismo nivel que la actuación especifica de los sistemas (incluido el de Servicios Sociales comunitarios).

Todo ello ha traído consigo el establecimiento de modelos de organización alternativos en distintos centros de Servicios Sociales comunitarios, inmersos en planes comunitarios integrales. Las nuevas formas tratan de superar la organización clásica, para reordenar la vida del centro desde esquemas de intervención globalizada ( sin renunciar con ello a su naturaleza prestadora). En la ciudad objeto de estudio, esta renovación organizativa se ha producido en dos centros ubicados en sendos barrios desfavorecidos. Los nuevos modelos organizativos se han guiado por el criterio: diferenciar, sin separar. En lugar de la estructuración clásica en cuatro servicios relativamente autónomos, los centros se han estructurado del siguiente modo:

1) Dos niveles de actuación de conjunto diferenciados. Uno, de intervención ligera dedicada a demandas cuya gestión puede agilizarse. El otro, de intervención de acompañamiento pensada para actuaciones más complejas en la que se trabaja en procesos prolongados. La mayor parte del equipo profesional ha sido adscrito a este último nivel.

2) Estableciendo, para el segundo, un conjunto de áreas interconectadas que con un enfoque integrado, abordan los tres grandes procesos carenciales de intervención social (Aguilar,

${ }^{8}$ Un ejemplo ilustrativo puede encontrarse en la Plataforma Nosotros también somos Sevilla que agrupa a diferentes organizaciones vecinales del Polígono Sur de Sevilla y lleva varios años reivindicando un modelo de intervención unificado para el conjunto de recursos dedicados al bienestar en este barrio. 
2009): los relacionados con déficits de autonomía, los programas dirigidos a la inclusión social y los que atienden a las dificultades para la convivencia. Esta diferenciación operativa se ha compaginado con espacios de trabajo de conjunto dentro del centro, y con espacios de trabajo en red externos, posibilitadores del encuentro con otros actores con los que se comparte intervención.

\section{Nuevas formas, nuevos retos}

A nadie se le escapa que este nuevo escenario, más complejo, obliga a la gente de Servicios Sociales comunitarios a resituar sus formas de presencia, su acción. Se relega la presencia monodialógica anterior, en la que cada cual se encargaba de su parcela (aun existiendo cierta coordinación formal), y la agenda interventora viene cada vez más marcada por lo que se planifica en espacios más transversales y trabados. Se toman muchas decisiones en mesas en las que otros actores opinan sobre lo que antes les era propio, al tiempo que se pide a los Servicios Sociales comunitarios orientaciones que nutran de criterios y pistas para el hacer de terceros (sociosanitarias, socioeducativas, etc.).

Además, el liderazgo de estas redes es más dinámico. Los planes comunitarios integrales se han ido ordenando en diferentes ejes operativos (urbanismo, bienestar social y economía y empleo, por ejemplo), con la idea fomentar un conjunto de redes por eje integradas en una red global en lo local (la del conjunto de entidades partícipes de los planes comunitarios integrales). Cada uno de los ejes agrupa a las diferentes instituciones y entidades relacionadas con la temática (Servicios Sociales está encuadrado en el eje de bienestar social). Este renovado modo de acción ha generado con frecuencia dificultades de ubicación de estos dispositivos, dudas sobre cuál es su nuevo papel en la gobernanza de lo comunitario. En unos casos se han puesto al frente, dispuestos a liderar los procesos, como nodo activo que cataliza las relaciones en torno a la dimensión social del proyecto de desarrollo9. En otros este liderazgo ha sido cedido a otras instituciones, incluso a entidades del Tercer Sector ${ }^{10}$.
Un último reto tiene que ver con el proyecto de intervención que lleva tras de sí el criterio de gobernanza. Es sabido que las mutaciones que han afectado al Estado de bienestar (más intensas en el momento presente), han reemplazado en política social el concepto de gobierno por el de gobernanza. Este cambio significa, más o menos, que lo que antes era un monólogo (del Estado) con un conjunto de actores figurantes (mercado y Tercer Sector), hoy se convierte en una representación coral. Sin embargo estas nuevas formas no son ni mucho menos uniformes en el ámbito de lo social-local.

- En unos casos tal gobernanza se entiende principalmente como vía de eficiencia. Interesa, por lo tanto, implicar en la misma únicamente a aquellas entidades que aporten algo que ayude a la mejora de la gestión, quienes puedan echar una mano a lo que se hace desde lo público.

- En el otro extremo, se percibe la gobernanza como vía de ciudadanía, como ocasión para fortalecer el debilitado proyecto cívico. Desde este segundo, el patrón el valor participación se sitúa por delante del valor gestión. Interesa fomentar procesos de gobernanza porque, más allá de las mejoras en la gestión de la intervención (que también se producen), son un cauce para fortalecer la capitalización social del territorio. Esta segunda concepción supone, retomando las intenciones fundadoras de Servicios Sociales comunitarios, un medio y al mismo tiempo un fin para estos dispositivos.

Estas son - a mi juicio- la dos tesituras estratégicas que en el presente han de afrontar los Servicios Sociales en este tipo de dinámicas integrales y reticulares. Nadie cuestiona la necesidad del trabajo en redes, pero está por decantar el tipo de rol que han de asumir los Servicios Sociales comunitarios en las mismas, así como el enfoque que se va a imprimir a sus formas de gobernanza.

\section{Dos lógicas en busca de dialógica}

En el trasfondo del recorrido de conjunto descrito hasta el momento subyacen dos tipos di-

\footnotetext{
${ }^{9}$ Un ejemplo puede encontrarse en la ZNTS de Tres Barrios en Sevilla.

${ }^{10}$ Un ejemplo puede encontrarte en la ZNTS Distrito $V$ de Huelva donde el liderazgo corresponde a una Fundación sin ánimo de lucro.
} 
ferente de aproximación a lo comunitario por los Servicios Sociales, cada uno lleva consigo su modelo de intervención social. Me detengo en este apartado en la descripción de las dol lógicas, para tratar después de provocar un diálogo entre ambas.

\subsection{Aproximación 1. La «comunidad como objeto»}

En la primera de las concepciones, se entiende la comunidad como un objeto interventor, una geografia humana que delimita un marco de acción. La misión de los Servicios Sociales frente a tal objeto será facilitar el acceso de la población a una oferta de bienes (ajustados lógicamente a unas necesidades predefinidas por las instancias tecno-políticas), concretados en prestaciones y recursos. La vía ideal de acceso será el derecho subjetivo; los Servicios Sociales comunitarios se consolidan institucionalmente en la medida en que tiene capacidad para elevar a esta categoría el conjunto de bienes que otorga.

A efectos prácticos este modelo, con fuerte inspiración en la idea de community care $^{11}$, apuesta por concretar el conjunto de dinamismos y energías institucionales que operan en $l o$ comunitario en torno a una oferta específica de servicios personales. Tales servicios quedan organizados como asistencia en la comunidad más que como asistencia de la comunidad (Herrera, 1998), de modo que los sujetos pierden peso para reconvertirse en beneficiarios objeto.

En este escenario los Servicios Sociales comunitarios precisarán principalmente: 1) una oferta de programas de atención; 2) unos sistemas de organización profesional y laboral; y 3 ) unas metodologías y procedimientos de actuación (Gómez et al: 2004).

\subsection{Aproximación 2. La «comunidad como sujeto»"}

Desde este segundo tipo de aproximaciones, se considera la comunidad sobre todo como una textura relacional (Schütz, 2003), dotada de sus propios dinamismos y capacidades. Más allá de los criterios de espacio y proximidad, el modelo de intervención social se sostiene sobre la capacidad de la cooperación horizontal entre actores y sujetos. Sousa Santos (2005, p. 311) lo llama el principio de comunidad, entendiendo que tal dinamismo cooperativo funciona en la intervención en interconexión con otro principio, el de Estado (basado en la obligación política vertical) ${ }^{12}$.

Se conciben, así, los Servicios Sociales como parte del entramado de bienes colectivos que actúan como promotores de la calidad de vida de la comunidad. Por lo que, más allá de su función facilitadora, se entiende que estos dispositivos están llamados a contribuir a mejorar el arraigo de los sujetos al entramado relacional. La vía ideal para ello será el empoderamiento, entendido como incremento del capital social del territorio (Vidal, 2009).

En este escenario los Servicios Sociales comunitarios precisarán principalmente unos instrumentos de mediación consistentes y la capacidad para establecer procesos de acompañamiento que favorezcan la inclusión de tales sujetos en una doble dimensión individualcolectiva.

\subsubsection{Una aproximación dialógica}

Con frecuencia se consideran las dos representaciones detalladas en este apartado como vías separadas, lo que hace que el discurso sobre la renovación discurra a menudo por dos trayectos estratégicos diferenciados (Barbero, 2010, p. 69): uno orientado a la modificación del dispositivo institucional y el otro al sostenimiento de abordajes colectivos. Sin embargo, el discurso profesional, en espacios como los barrios vulnerables, clama por la necesidad de impulsar a un tiempo ambos trayectos. Esta demanda obliga a una dialógica objeto y sujeto en la presencia comunitaria, en donde los Servicios Sociales comunitarios compaginan su rol de facilitadores de bienes que posibilitan el acceso a un grado razonable de autonomía en los sujetos; con el arraigo en torno al entramado rela-

\footnotetext{
${ }^{11}$ Esta forma de política social, de inspiración anglosajona, aboga por el desarrollo de servicios sociosanitarios en lo local. Herrera define la community care como: todas aquellas formas de asistencia (incluida la asistencia residencial) que puedan ser elaboradas por los servicios sanitarios y sociales de base de los entes locales (1998, p. 254).

12 Sousa Santos alude también a un tercer principio, el de mercado, de menor relevancia en este caso.
} 


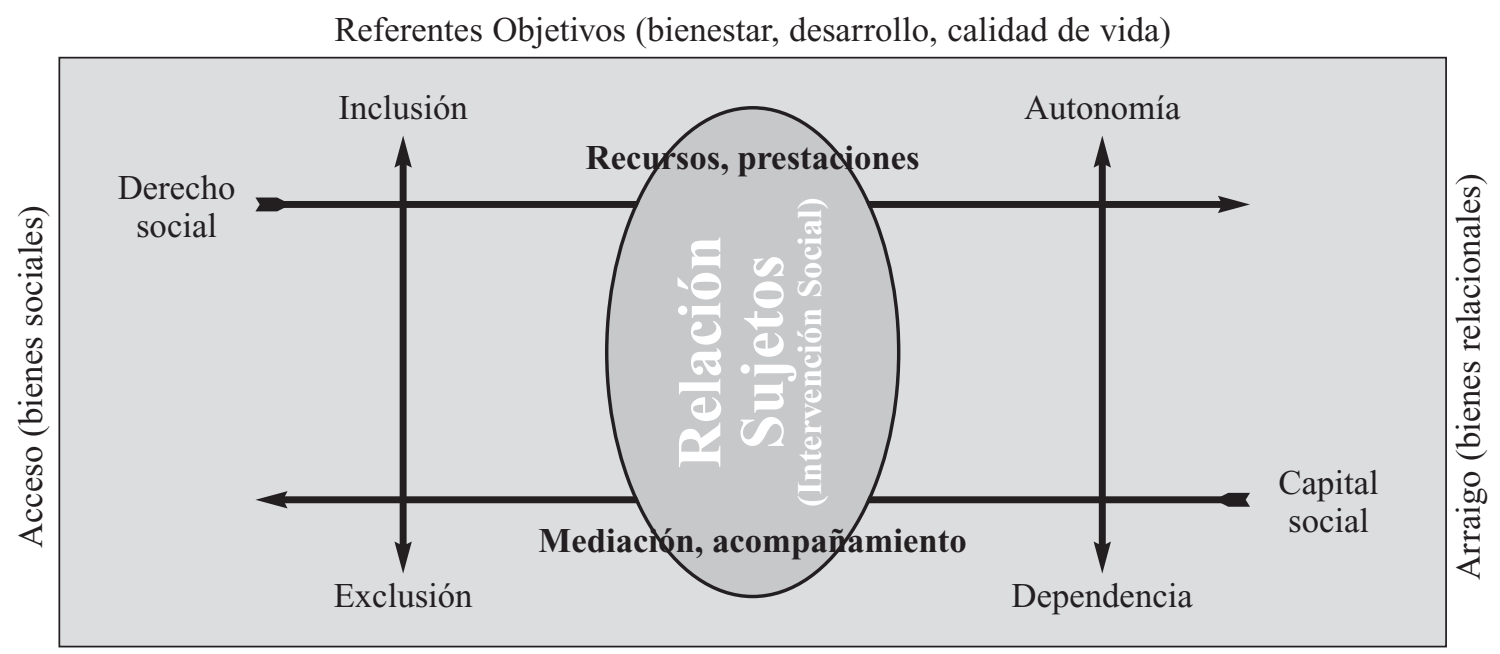

Realidad Objeto (necesidadedes, problemas, riesgos sociales)

Gráfico 1. Dialógica objeto y sujeto. Fuente: Elaboración propia.

cional que favorece la inclusión normalizadora. Esta incardinación dialógica podría quedar expresada del siguiente modo (gráfico 1).

\section{Pistas de renovación desde el discurso profesional. Recuperar el Trabajo Social en Servicios Sociales}

El anterior esquema trata de poner en orden el conjunto de valores, criterios y procesos que los profesionales de estos centros, tras profundos debates, entienden como vías idóneas para el despliegue de los Servicios Sociales comunitarios. Frecuentemente el último tipo de interrogantes que ha surgido en estos espacios de discusión atendía a las orientaciones para avanzar hacia este enfoque dialógico y al tipo de prácticas a impulsar. Los aportes se agrupan aquí sobre cuatro clusters, que ahora presentaré de modo sintético. Sin embargo, por encima de ellos aparece un factor que hilvana el conjunto de propuestas: los profesionales coinciden en la necesidad de recuperar el Trabajo Social como soporte de la intervención. El siguiente discurso fundamenta esta idea:

Para ser un buen profesional en los comunitarios no hace falta saber mucho trabajo social. Un buen profesional se mide aquí por la capacidad agilizar la gestión de los casos. Los expedientes queman en las manos, quien tiene habilidad para cerrar pronto un caso es un buen profesional. En el fondo nadie se preocupa por cómo trabajas, por lo que puedas lograr con la gente, nadie te pregunta por el trabajo social que haces.

En realidad no se puede hablar de renovar el enfoque comunitario sin poner esta cuestión en la mesa, y por ese motivo el conjunto de propuestas prácticas se refieren en el fondo a recuperar la relevancia del Trabajo Social en la construcción de los Servicios Sociales comunitarios. Cuando apunto este hecho, no me estoy refiriendo a la dimensión corporativa, sino a una concepción disciplinar en sentido amplio que entienda que el trabajo social aporta un método con capacidad de articular con otra lógica los Servicios Sociales comunitarios. Desde esta idea de conjunto adquieren sentido los señalados cuatro ámbitos de sugerencia práctica:

El primero de ellos se refiere a la relación con los sujetos. Las aportaciones se orientan de forma general hacia la idea de mejorar la relación de ayuda. El aporte más repetido se refiere a la necesidad de refuerzo de las dinámicas de acompañamiento (trabajo en itinerarios). Un segundo aspecto en este cluster se concreta en la superación del enfoque exclusivamente individualizador mediante formas de trabajo con grupos de afectados (abordaje de elementos de autoorganización). Otras aportaciones tratan cuestiones como el diseño del espacio físico de los centros, pensados como lugares de racionalidad administrativa (casi todo despachos), y se apunta la necesi- 
dad de rediseño con criterios de acogida y encuentro.

El establecimiento de alianzas consistentes aparece como un segundo bloque de propuestas, el conjunto de las mismas se concentra principalmente en la alianza con los dispositivos de ámbito laboral y económico para la promoción de estrategias de inclusión, con los de ámbito socio-educativo para la intervención en línea de convivencia e integración y con el socio-sanitario para las cuestiones relativas a la promoción de la autonomía funcional.

Las formas de gestión interna de los centros ocupan el tercer grupo de prácticas renovadoras. Todas ellas van en la línea de fortalecimiento de los espacios más trabados y participados en los procesos de gestión (formación, planificación, evaluación). Se plantea orientar en esta lógica más participada las dinámicas de gestión del conocimiento y la calidad.

La conexión con lo cívico ordena el cuarto grupo de propuestas. Aparecen aquí dos líneas de mejora: la primera agrupa propuestas prácticas normalizadoras que permitan aproximar a los centros de Servicios Sociales comunitarios a la cotidianidad de los barrios (ubicación en equipamientos polivalentes, mayor incardinación con equipamientos cívicos, presencia en acontecimientos significativos del barrio, etc.).
La segunda se refiere al trabajo en red, y las propuestas van orientadas a la revisión de los sistemas de planificación (rediseñar los planes de centro y abrirlos a los aportes de otras entidades sociales), o a la mejora de la implicación de los equipos profesionales en los espacios de trabajo comunitario.

\section{A modo de conclusión}

En los Servicios Sociales objeto de este estudio ha primado un modelo de intervención asentado en prácticas en las que la comunidad es tratada como objeto sobre el que aplicar acciones de naturaleza facilitadora; relegándose a un segundo plano la intervención de lógica empoderadora y activadora, que concibe al barrio y sus vecinos como sujetos del propio proceso de acceso al bienestar.

Los profesionales son conscientes de las limitaciones que, en este tipo de espacio vulnerable, imprime este hecho para el logro de dinamismos inclusivos. Abogan de forma mayoritaria por la necesidad de renovación del enfoque de intervención de Servicios Sociales comunitarios, entendiendo que tal renovación habría de posibilitar un mayor equilibrio entre las prácticas de garantía de bienes sociales y las de acompañamiento y mediación activadora.

\section{Referencias bibliográficas}

Aliena Miralles, R. (2011). Pobreza y sujeto. Revista Española del Tercer Sector, 16, 47-78. Madrid: Fundación Luis Vives.

Aguilar Hendrickson, M. (2009). Servicios Sociales: Las tribulaciones de un sector emergente. En L. Moreno (ed.). Reformas de las politicas de Bienestar en España (171-205). Madrid: Siglo XXI

Arriba González de Durana, A. (2008). Políticas sociales y bienes sociales. Procesos de vulnerabilidad y exclusión social. Madrid: Cáritas-FOESSA.

Barbero, J. M. (2010). Retos metodológicos en trabajo social comunitario. En Redefiniendo el trabajo comunitario. Vitoria-Gasteiz: Escuela de Trabajo Social de la Universidad del País Vasco.

Casado, D. y Fantova, F (coords.) (2007). Perfeccionamiento de los Servicios Sociales en España. Informe de la Ley sobre autonomía y dependencia. Madrid: FOESSA.

Colectivo IOE. (1990). Ideologías de la intervención social en la España de los 90. En Documentación Social, 81, 53-84. Madrid: Cáritas Española.

Consejo General de Colegios de Diplomados en Trabajo Social (1986). Encuentro sobre servicios sociales comunitarios. Madrid: Siglo XXI.

Fombuena Valero, J. (2000). Pureza y mestizaje en Trabajo Social. Revista de Trevall Social 158, 8-33. Barcelona: Colegio Oficial de Diplomados en Trabajo Social.

García Roca, J. (2006). Memorias silenciadas en la construcción de servicios sociales. Cuadernos de Trabajo Social, 19, 197-212. Madrid: Universidad Complutense.

Gómez, A., Rosa, M. y Ruiz, E. (2004). Transformación organizacional y prestación de servicios. Investigación participativa en un Centro de Servicios Sociales Comunitarios. Sociología del Trabajo, 51, 107-128. Madrid: Siglo XXI. 
Herrera Gómez. M. (1998). Nuevos desafíos en políticas sociales: La «community care». REIS, 82, 249-281. Madrid: CIS.

Jaraíz Arroyo, G. (2004). Desarrollo local, barrios desfavorecidos y cohesión urbana. Documentación Social, 133, 127-152. Madrid: Cáritas Española.

Malagón Bernal, J.L. (1999). Fundamentos del trabajo social comunitario. Sevilla: Aconcagua.

Marchioni, M. (1966). Algunos elementos teóricos sobre desarrollo comunitario. Documentación Social, 1, 5-19. Madrid: Cáritas Española.

Renes Ayala, V., Fuentes Rey, P., Ruíz Ballesteros, E. y Jaraíz Arroyo, G. (2007). Realidad, pensamiento e intervención Social. Documentación Social, 145, 11-36. Madrid: Cáritas Española.

Schutz, A (2003). El sentido común y la interpretación científica de la acción humana. En El problema de la realidad social. Buenos Aires: Amorrortu.

Sousa Santos, B. (2005). El milenio huérfano. Ensayos para una nueva cultura política. Madrid: Trotta.

Subirats, J. (dir.) (2007). Los Servicios Sociales de Atención Primaria ante el cambio social. Madrid: Ministerio de Trabajo y Asuntos Sociales.

Vidal Fernández, F. (2009). Pan y Rosas. Fundamentos de exclusión social y empoderamiento. Madrid: FOESSA.

Vila Mancebo, A. (2010). Tendencias en la nueva legislación de Servicios Sociales. Madrid: EAPN. 